\title{
Surface morphology and wettability for thin films of beta-iron disilicide produced through direct-current sputtering utilizing a pair of facing targets
}

\author{
Peerasil Charoenyuenyao ${ }^{1}$, Nathaporn Promros ${ }^{1, *}$, Rawiwan Chaleawpong ${ }^{1}$ and Tsuyoshi Yoshitake $^{2}$ \\ ${ }^{1}$ Department of Physics, Faculty of Science, King Mongkul's Institute of Technology Ladkrabang, Bangkok 10520 \\ ${ }^{2}$ Thailand Department of Applied Science for Electronic and Materials, Kyushu University, Fukuoka 816-8580, Japan
}

\begin{abstract}
In the current work, beta-FeSi $i_{2}$ films were epitaxially produced onto $\mathrm{Si}(111)$ wafer substrates via usage of facing-targets direct-current sputtering (FTDCS). The temperature for substrate heating was maintained at $600{ }^{\circ} \mathrm{C}$ and the sputtering pressure was set at $1.33 \times 10^{-1} \mathrm{~Pa}$. The surface morphology and contact angles of the beta- $\mathrm{FeSi}_{2}$ films were explored consistently in this research. Images of threedimensional AFM and FESEM for the beta-FeSi ${ }_{2}$ film surface revealed a smooth surface with a root mean square roughness of $1.31 \mathrm{~nm}$ and a porous area. The average contact angle between the dropped water and beta-FeSi $i_{2}$ film surface was found to be $98.7^{\circ}$, establishing that the surface of the beta-FeSi $i_{2}$ films was hydrophobic. The acquired experimental results revealed the commencement of the hydrophobic surface feature of the beta-FeSi $i_{2}$ films produced via FTDCS approach.
\end{abstract}

\section{Introduction}

Over the past few years, iron disilicide $\left(\mathrm{FeSi}_{2}\right)$ possessing an orthorhombic semiconducting phase (beta) has garnered considerable attention from numerous researchers as a result of its suitable semiconducting features $[1,2]$. It has been experimentally supported that it is possible to be epitaxially produced onto Si layers. The lattice mismatches for epitaxial production between films of beta- $\mathrm{FeSi}_{2}$ and $\mathrm{Si}$ layers were from 2 to 5 percent $[3,4]$. Further, the compound beta-FeSi ${ }_{2}$ was produced from silicon and iron, which are environmentally friendly, non-toxic, and plentiful in nature [5]. Beta-FeSi $i_{2}$ holds direct (0.85 electron volt) and indirect ( 0.76 electron volt) optical band gaps as well as an absorption coefficient $>10^{5} \mathrm{~cm}^{-1}$ at 1.2 electron volt [6-8]. It has been evidentially confirmed by previous research that the beta- $\mathrm{FeSi}_{2}$ film surface was quite smooth [9]. These features of beta-FeSi $\mathrm{F}_{2}$ are appropriate for usage in optoelectronic devices and superhydrophobic surfaces. Employing facing-targets direct-current sputtering (FTDCS), the production of beta-FeSi $i_{2}$ films onto $\mathrm{Si}(111)$ wafers was achieved at low temperatures for substrate heating in the previous study [9]. The FTDCS approach consists of a pair of targets set in a parallel direction. Particularly, the substrate is situated outside of the plasma area and vertical with the pair of targets. In comparison to common radio-frequency sputtering, this approach has the following advantages: (a) sputtering at low pressure operation, (b) higher density of plasma created from sputtering, (c) less increment in substrate temperature, (d) less damage and (e) obtained films with low difference stoichiometry in comparison with a target as a result of the substrate being free of plasma [4, 9]. Additionally, the energy of particles that reached the substrate surface was higher compared to utilization of different approaches because of sputtering at low pressure operation $[4,9]$. Based on the merits of FTDCS, the epitaxial production of beta- $\mathrm{FeSi}_{2}$ thin films onto the $\mathrm{Si}(111)$ was accomplished when the temperature for substrate heating was $600{ }^{\circ} \mathrm{C}$. The thin films of beta$\mathrm{FeSi}_{2}$ created in this work presented n-type property as well as carrier density of approximately $5 \times 10^{17} \mathrm{~cm}^{-3}$ [9]. The produced hetero-structure between p-type $\mathrm{Si}$ wafers and n-type beta-FeSi $i_{2}$ thin films can be utilized in photovoltaic and photodiode applications [10, 11]. Despite the face beta-FeSi $i_{2}$ possesses desirable properties for hydrophobic surface applications, there have been few studies or reports on the assessment of wettability properties of beta-FeSi $i_{2}$ films produced via FTDCS for employment in such applications. Consequently, the current research proposes study of their surface morphology as well as wettability behavior. It was anticipated that the average contact angle between the dropped water and the beta-FeSi ${ }_{2}$ film surface would be greater than $90^{\circ}$ (hydrophobic surface). According to all available knowledge and information, this research comprises the first exploration and report concerning wettability based on beta-FeSi $i_{2}$ thin films produced through FTDCS.

${ }^{*}$ Corresponding author: nathaporn promros@,kyudai.jp 


\section{Experimental details}

Based on the usage of FTDCS, a $300 \mathrm{~nm}$ beta-FeSi layer was produced onto a wafer of p-type $\mathrm{Si}(111)$ by employing a pair of $\mathrm{FeSi}_{2}$ alloy targets. The temperature for substrate heating was fixed at $600{ }^{\circ} \mathrm{C}$ during film production. Before epitaxial production, the organic and oil layers were eliminated from the p-type $\operatorname{Si}(111)$ wafer by rinsing in acetone, methanol, and deionized water for five minutes in each step. Next, a diluted hydrofluoric (HF) acid solution (concentration of 1 percent) was applied to eradicate the existing oxide layer. The HF residue was purged instantaneously from the p-type $\mathrm{Si}$ wafer in deionized water. After cleaning, the prepared $\mathrm{Si}$ wafer was attached to the substrate holder inside the FTDCS chamber, where the space between the Si wafer substrate and $\mathrm{FeSi}_{2}$ targets was set at $7.5 \mathrm{~cm}$. The pressure inside of the chamber was evacuated to $3 \times 10^{-5} \mathrm{~Pa}$ using both a turbomolecular pump and rotary pump. The sputtering process was operated in argon gas (purity: $6 \mathrm{~N}$ ). The flow rate of the argon gas and pressure of sputtering were maintained at $15 \mathrm{sccm}$ and $1.33 \times 10^{-1} \mathrm{~Pa}$, respectively. The applied voltage for sputtering was assigned to $1 \mathrm{kV}$, while the generated sputtering current was about $1.5 \mathrm{~mA}$. Thorough study of X-ray diffraction (XRD) for the beta- $\mathrm{FeSi}_{2}$ layer epitaxially produced on $\mathrm{Si}(111)$ wafers was performed in a Rigaku RINT 2000/PC. The surface morphology view of beta-FeSi $i_{2}$ films was explored using FESEM (Carl Zeiss Auriga Field Emission Scanning Electron Microscope). The roughness of the film surface was inspected by means of an XE-120 Atomic Force Microscope (AFM). The wettability of the film surface was determined using an OCA 20 measuring instrument.

\section{Results and discussion}

Figure 1(a) presents the scanned XRD pattern of the produced thin films of beta-FeSi $i_{2}$ onto the layer of $\operatorname{Si}(111)$ wafers. Measurement was executed utilizing a mode of $2 \theta-\theta$ scan. From the appearance pattern, weak 404/440 and intense 202/220 peaks could be found. These peaks are essential for beta- $\mathrm{FeSi}_{2}$ films, which can be epitaxially produced on $\mathrm{Si}(111)$. In order to measure the pole figure, a specific crystallographic plane of the $2 \theta-\theta$ scan should be fixed, which determines the Miller indices of the films. From the XRD pattern, the beta-202/220 peak was close to the strong peak of the Si-111 plane. Namely, the obtained pole figure image, which was measured in this plane, might show the pole figure of the beta-202/220 peak with interaction from the substrate plane. Therefore, the diffraction peak of beta-404/440 was chosen as the main scanning plane in this XRD pole figure measurement. In the measurement process, the pole figure was performed by varying two geometrical parameters, such as the tilt angle $(\Psi)$ from beta- $\mathrm{FeSi}_{2}$ film surface normal direction and the rotation angle $(\phi)$ around the surface of the films in the normal direction. Figure 1(b) depicts the acquired pattern of pole-figure concerning the peak of beta-404/440 for the beta- $\mathrm{FeSi}_{2}$ films produced onto $\mathrm{Si}(111)$ wafers. This result reveals the occurrence of 3 kinds of epitaxial variants. The rotation angle was 120 degrees to each other [3].

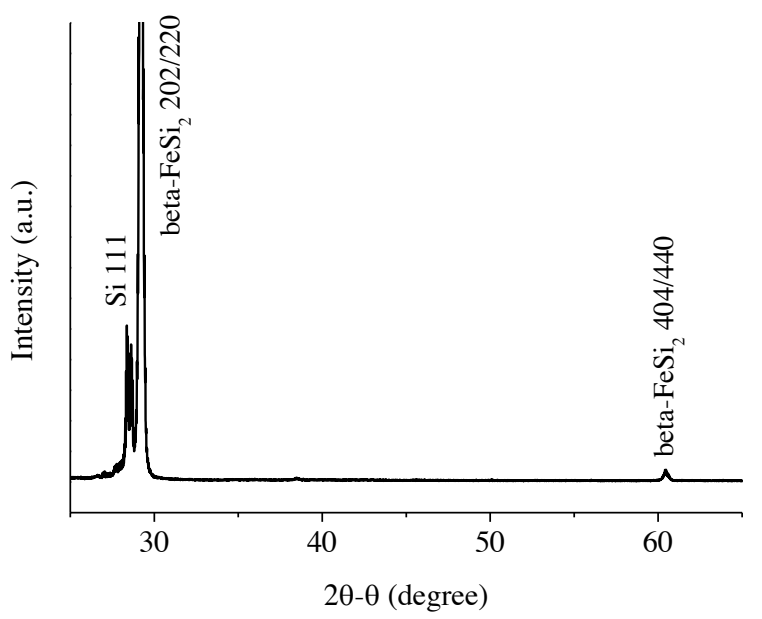

(a)

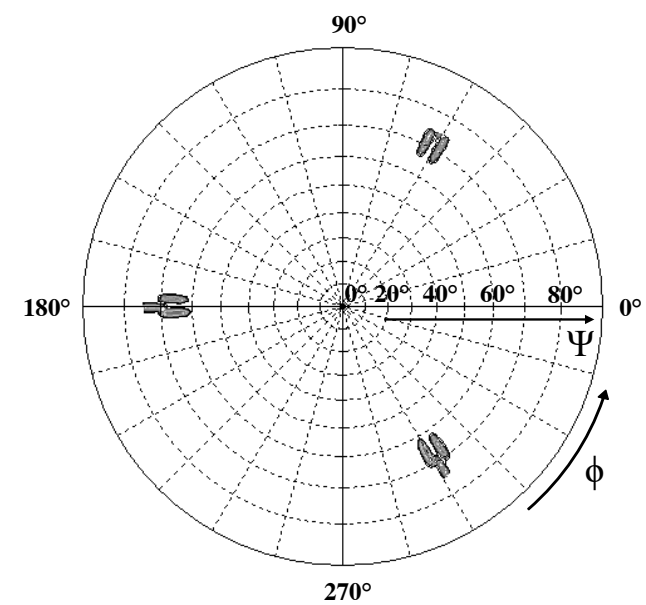

(b)

Fig. 1. (a) The XRD pattern measured in a mode of $2 \theta-\theta$ scan and (b) the pole-figure pattern of beta- $\mathrm{FeSi}_{2}$ films produced onto $\mathrm{Si}(111)$ wafers.

Figure 2(a) illustrates a FESEM micrograph with 50k magnitude in plain surface view of the produced beta-FeSi $i_{2}$ film. It was observed that a large amount of crystallites with small sizes covered the film surface. Further, the produced beta- $\mathrm{FeSi}_{2}$ films contained many grain boundaries and a porous area that existed at the edge of the grain boundary. In Figure 2(b), a crosssectional FESEM micrograph is presented showing a layer of beta- $\mathrm{FeSi}_{2}$ thin film coated onto a layer of $\mathrm{Si}(111)$ wafer. In the cross-section micrograph, the beta-FeSi $i_{2}$ film surface produced through FTDCS was level and appeared non-fractured along the path. 


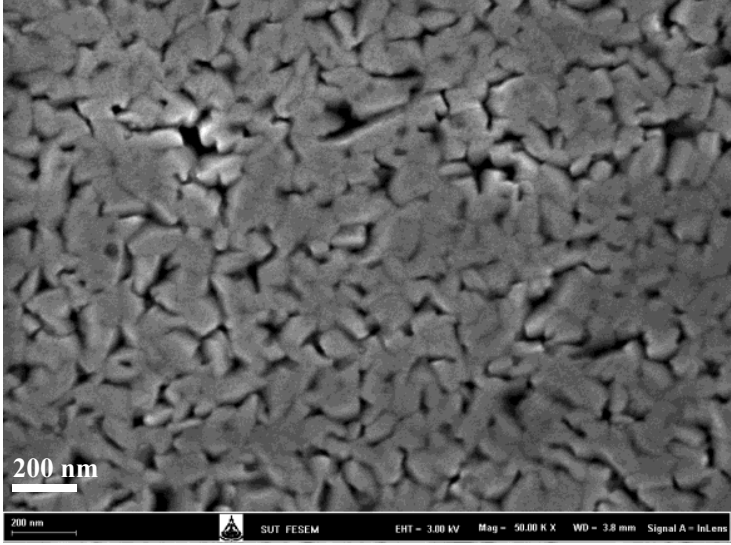

(a)
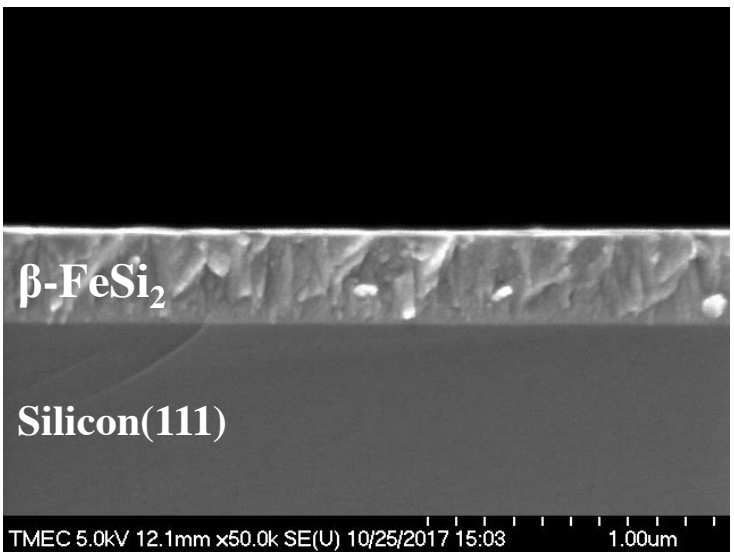

(b)

Fig. 2. FESEM micrograph in (a) plain view and (b) crosssectional view for the layer of beta-FeSi $\mathrm{F}_{2}$ films produced onto the layer of $\mathrm{Si}$ (111) wafer.

A three-dimensional AFM image of beta-FeSi ${ }_{2}$ films, scanning in non-contact mode, is illustrated in Figure. 3. The scanning result demonstrates the rather smooth surface of the beta-FeSi $\mathrm{Film}_{2}$. The estimated root mean square roughness $\left(\mathrm{R}_{\mathrm{rms}}\right)$ of the film surface was $1.31 \mathrm{~nm}$. Some pinholes are observable within the scanning area of $500 \times 500 \mathrm{~nm}$, which conform to FESEM surface images. This can be represented by the fact that, in the production process of beta- $\mathrm{FeSi}_{2}$ films by usage of FTDCS, the Si substrate is located on a substrate holder far away from the plasma that was generated from sputtering $[4,9]$. Furthermore, the temperature of the produced beta- $\mathrm{FeSi}_{2}$ layer surface did not increase as a result of the $\mathrm{Si}$ substrate being arranged far from the plasma region. Based on this situation, the surface of the produced beta-FeSi ${ }_{2}$ layer suffered minimal damage from the destruction of sputtering plasma $[4,9]$.

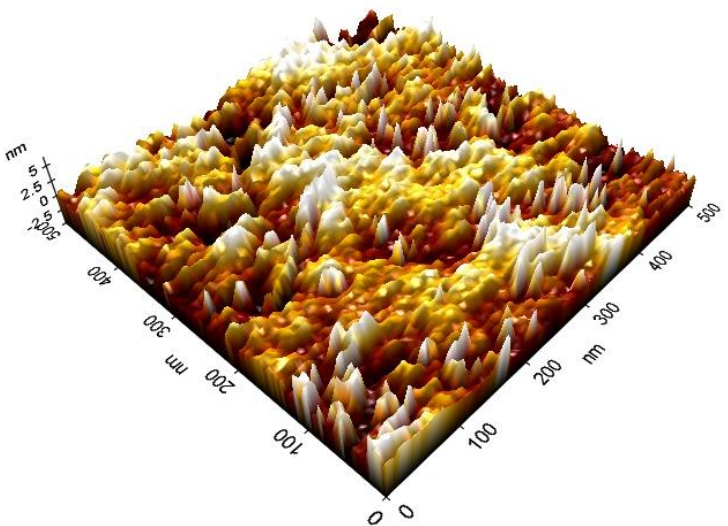

Fig. 3. Three-dimensional AFM image of beta-FeSi ${ }_{2}$ films produced by FTDCS.

In order to expose the wettability behavior of the beta$\mathrm{FeSi}_{2}$ thin films produced by means of FTDCS in this study, the contact angle between the beta-FeSi $i_{2}$ film surface and the dropped water was measured. Figure 4 presents an image of the measured contact angle between film surface and water droplet. The average contact angle between the water droplet and film layer was $98.7^{\circ}$ $\left(99.0^{\circ}\right.$ and $98.4^{\circ}$ for the left and right sides of contact angle, respectively). From the obtained results, the surface of the beta-FeSi $i_{2}$ films exhibited hydrophobic properties. Generally, a hydrophobic surface is labeled as having contact angles in the angle range of $90^{\circ}<\theta<$ $150^{\circ}$ [12-14]. According to the FESEM image, the beta$\mathrm{FeSi}_{2}$ film surface has an occurrence of porous territory. This can be explained by the wetting model of CassieBaxter, which describes the wetting phenomena of the porous area underneath liquid drops. The porous area is an act to the air pocket, where air can infiltrate into the grooves and support the lifting of dripped liquid, resulting in a higher contact angle than for a flat surface [15-17].

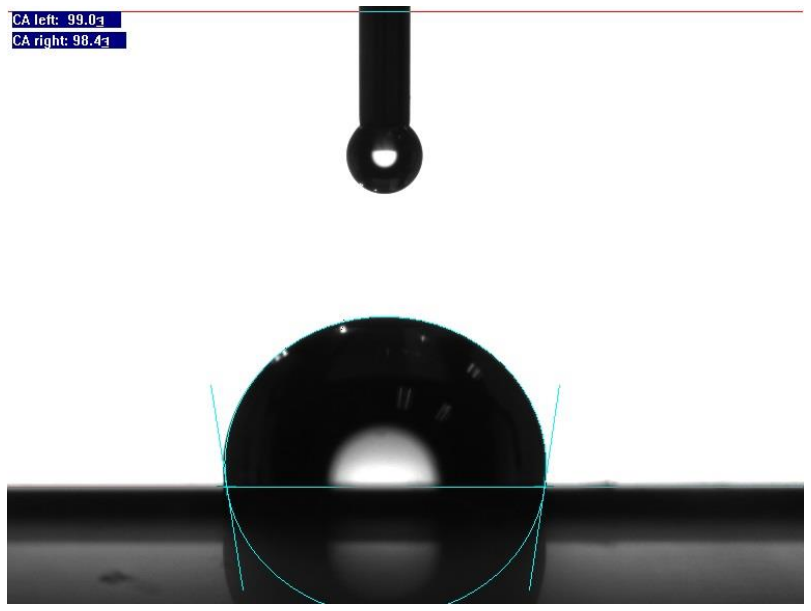

Fig. 4. The contact angle between the water droplet and plane of beta-FeSi $i_{2}$ layer produced on $\mathrm{Si}(111)$ wafer via FTDCS.

\section{Conclusions}

In the present work, a layer of beta-FeSi ${ }_{2}$ was produced onto a $\mathrm{Si}(111)$ wafer via FTDCS. The temperature for 
substrate heating and the sputtering pressure were maintained at $600{ }^{\circ} \mathrm{C}$ and $1.33 \times 10^{-1} \mathrm{~Pa}$, respectively. Surface morphology for the produced beta- $\mathrm{FeSi}_{2}$ films was explored using FESEM and three-dimensional AFM images, which demonstrated the existence of a porous area (pinholes) and $\mathrm{R}_{\mathrm{rms}}$ of $1.31 \mathrm{~nm}$. The average contact angle of the produced beta- $\mathrm{FeSi}_{2}$ film surface was $98.7^{\circ}$. This proved the hydrophobic behavior of the film surface. From the acquired results, it was revealed that beta-FeSi $i_{2}$ films produced via FTDCS could potentially be applied to hydrophobic surfaces.

The present study was supported by a research grant from the AUN/SEED-Net as a CRA project as well as by the Faculty of Science (KMITL).

\section{References}

1. D. Leong, M. Harry, K.J. Reeson, K.P. Homewood, Nature 387, 686 (1997)

2. T. Sunohara, K. Kobayashi, T. Suemasu, Thin Solid Films 508, 371 (2006)

3. M. Shaban, K. Nomoto, S. Izumi, T. Yoshitake Appl. Phys. Lett. 94, 222113 (2009)

4. N. Promros, R. Baba, M. Takahara, T.M. Mostafa, P. Sittimart, M. Shaban, T. Yoshitake, Jpn. J. Appl. Phys. 55, $06 \mathrm{HC} 03$ (2016)

5. M. Shaban, S. Izumi, K. Nomoto, T. Yoshitake, Appl. Phys. Lett. 95, 162102 (2009)

6. N. Promros, K. Yamashita, R. Iwasaki, T. Yoshitake, Jpn. J. Appl. Phys. 51, 108006 (2012)

7. M.C. Bost, J.E. Mahan, J. Appl. Phys. 64, 2034 (1988)

8. B. Tatar, K. Kutlu, M. Ürgen, Thin Solid Films 516, 13 (2007)

9. T. Yoshitake, Y. Inokuchi, A. Yuri, K. Nagayama, Appl. Phys. Lett. 88, 182104 (2006)

10. M. Shaban, K. Nakashima, T. Yoshitake, Jpn. J. Appl. Phys. 46, 7708 (2007)

11. S. Izumi, M. Shaban, N. Promros, K. Nomoto, T. Yoshitake, Appl. Phys. Lett. 102, 032107 (2013)

12. A. Kumar, G.K. Dalapati, H. Hidayat, F. Law, H.R. Tan, P.I. Windenborg, B. Hoex, C.C. Tan, D.Z. Chi, A.G. Aberle, RSC Adv. 3, 7733 (2013)

13. J. Drelich, E. Chibowski, D.D. Meng, K. Terpilowski, Soft Matter 7, 9804 (2011)

14. M. Krasowska, J. Zawala, K. Malysa, Adv. Colloid Interface Sci. 147-148, 155 (2009)

15. A. Lafuma, D. Quéré, Nat. Mater. 2, 457 (2003)

16. V. Mortazavi, M.M. Khonsari, Wear 372-373, 145 (2017)

17. A.M.A. Mohamed, A.M. Abdullah, N.A. Younan, Arab. J. Chem. 8, 749 (2015) 